\title{
Collaborative Development and Simulation of an Aircraft Hydraulic Actuator Model
}

\author{
Clément Coïc Johan Andreasson Anand Pitchaikani Johan Akesson Hemanth Sattenapalli \\ Modelon, \{name.surname\} @modelon.com
}

\begin{abstract}
This paper discusses the typical engineering workflow of an aircraft hydraulic actuator development enhanced using Modelon Hydraulics library and the new Modelon Impact environment. Modelon Impact consistent with Modelon's philosophy - enables having a more collaborative development and maintains more continuity between different phases of design cycle as well as democratizes models by making it available in different forms including dedicated apps.

In order to cover the scope of the engineering design workflow, several steps are discussed:

- Specification of the system requirements through test scenarios

- Sizing of the system based on a steady-state design point

- Re-use of the sized parameters for simulation of the model

- Performing design verification, based on dynamic simulation scenarios

- Export of the model using the Functional Mock-Up Interface standard, e.g, for cosimulation with flight control laws

As most realistic engineering workflows involve several teams and, therefore, several tools, this paper discusses the openness and connectivity of the proposed Modelon solution.
\end{abstract}

Keywords: $\quad$ Hydraulic actuation system, Model-based Design, Collaborative Design

\section{Introduction}

In a Model-Based System Engineering (MBSE) workflow, virtual prototypes (models) are used to facilitate the design and testing of the system under study. The terminology Model-Based Design (MBD) is also often used to emphasize that system sizing is performed with the help of models. While the complete MBSE design process would cover many types of different model, this paper focuses on the behavioral modeling of the system that is covered by the Modelica Language.

The Requirement, Functional, Logical, and Physical (RFLP) formalism would include additionally, requirement management, UML/SYSML models for the functional definition of the system or 3D/CFD/FEA models for the detailed physical design.

Figure 1 gives an overview of how Modelica models fits in the over-all design flow, using example applications from the Vehicle Dynamics Library: Starting from the left, lower fidelity models are used to explore large sets of potential configurations before gradually narrowing down the design. As the design work continues, the fidelity is increased. Beyond natively providing a large fidelity range, the models can also be evaluated against formal requirements (references to requirements library and doors?), connect to other models (FMI, external object), and/or reduced order models from tools for detailed physical design.

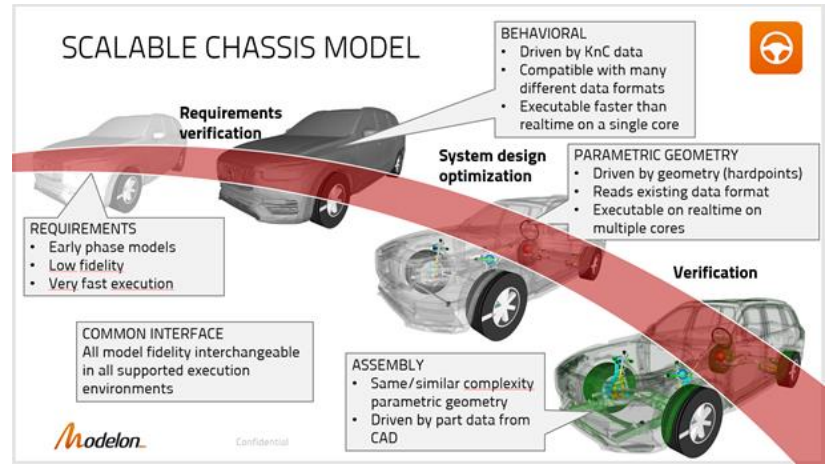

Figure 1. The Modelica Language allow for the design of scalable models, where fidelity can be adapted to the different stages of Modeli-Based Systems Engineering (MBSE).

The behavioral model-based design of a system can assist several tasks in the entire MBSE workflow. These are typically: system architecture definition, system presizing, component pre-sizing, detailed behavioral modeling and simulation, unitary and integration verification, real-time simulation for Hardware-in-theLoop (HiL) simulation [DRE14], etc.

Unfortunately, most of current engineering workflows are decoupled between these tasks. The main reason is often that each task is performed by a different person, often in a different team or company and the tools used are different.

Modelon's approach is to deploy Modelica, FMI and other open standards to promote collaboration. This is done in three ways: 
1) Modelon Library Suite ${ }^{1}$ is provided for capable Modelica tools. The suite consists of libraries covering several industries and applications. This enables models to be built, shared, and executed in collaborative efforts involving multiple tools.

2) The Optimica Compiler Toolkit ${ }^{2}$ is a Modelica engine for vendors that desire to include Modelica support in their offerings, and it is embedded in ANSYS Twin Builder ${ }^{3}$ and Siemens Simcenter Amesim ${ }^{\circledR}{ }^{4}$. On top of that they can add the Modelon Library Suite and/or libraries from other providers. In addition, Modelon's FMI tools ${ }^{5}$ provide key elements for linking together FMI-based toolchains.

3) Modelon Impact is our new end-user product that encapsulates our experience into one offering for collaborative product design. It combines the functionalities of our portfolio, including libraries and Modelica engine, with a web-based architecture and a novel user interface.

This paper discusses how Modelon provides streamlined MBSE workflows, connecting the broken streams. We will use, as an example, the development of an aircraft hydraulic actuator. The actuator is developed based on the Modelon Hydraulics Library that is available on multiple platforms.

The role of the aircraft actuator in his context is presented in Section 2. Section 3 describes the different phases of the model-based design of the actuator. Section 4 addresses each of these phases and emphasizes the collaborative aspects of our solution. A summary and conclusions are provided in Section 5.

\section{Aircraft hydraulic actuator}

\subsection{An aircraft hydraulic actuation system}

The actuation system of an aircraft performs a safety critical function that ensures controllability. Although today's trend is focused on developing electrical actuation systems, current aircrafts and new developments still require hydraulic fluid to power (most of) the actuators.

In this paper, the aircraft actuations system refers to the set of actuators, operated in closed-loop, that drives the control surfaces and the associated hydraulic systems. Assuming the use of hydraulic power, the main functions covered by the aircraft actuation systems are to [COI16]:

- Store hydraulic fluid, typically implemented by reservoirs, pressurized or non-pressurized.
- Generate hydraulic power - typically ensured by mechanical pumps, electric pumps, hand pumps, ram air turbine (RAT) and power transfer units (PTU).

- Store hydraulic power, mostly implemented by accumulators, and also by the capacitance effect of the lines.

- Distribute hydraulic power, mostly ensured by rigid lines and hoses as well as dedicated valves to distribute the fluid.

- Convert hydraulic power to mechanical power, typically performed by linear or rotary actuators. This function can be split into two main subfunctions:

- Meter hydraulic power, typically ensured by proportional control valves.

- Transform hydraulic power into mechanical power, usually performed by hydraulic cylinders (linear or rotary).

The example used throughout this paper is an aircraft hydraulic actuator that fulfills the functions "convert hydraulic power". In order to add realism to this paper, an A320-like aileron actuator is used as example.

\subsection{The role of the aileron actuator}

The roll control of an A320 aircraft is mainly driven by the aileron pitch angles, assisted by the external spoilers at low speed. Each aileron is actuated by two actuators. Each of these actuators is qualified as simplex, as each has only one hydraulic cylinder. The loss of the hydraulic power connected to one actuator would result in the loss of the actuator itself. Therefore, for a given aileron, both actuators are each fed by different hydraulic circuits to ensure redundancy.

The command and monitoring functions of the aileron actuators are realized by the Elevator and Aileron Computer (ELAC). In order to avoid forcefighting between the two actuators of an aileron and to reduce the overall wear and fatigue on the actuators, the ELAC commands one actuator to be "active" while the other is set to "stand-by".

Such an "active/stand-by" configuration is common on aircrafts. The active actuator drives the control surface while the stand-by one is driven by the surface motion - and generates some damping. In the event of the active actuator failure or loss, the ELAC would invert the roles so the surface could still be actuated by the functioning actuator

\footnotetext{
${ }^{1}$ https://www.modelon.com/products-services/modelon-library-suitemodelica-libraries/

${ }^{2}$ https://www.modelon.com/products-services/modelon-creatorsuite/optimica-compiler-toolkit/

${ }^{3}$ https://www.ansys.com/en/products/systems/ansys-twin-builder
}

\footnotetext{
${ }^{4}$ https://www.plm.automation.siemens.com/global/en/products/simcente r/simcenter-amesim.html

5 https://www.modelon.com/products-services/modelon-deploymentsuite/
} 


\subsection{Architecture description of the A320 aileron actuator}

Figure 2 [MAR18], depicts the architecture of an A320 aileron actuator. The numbering included in the description of the modes, below, are referring to the elements of this figure.

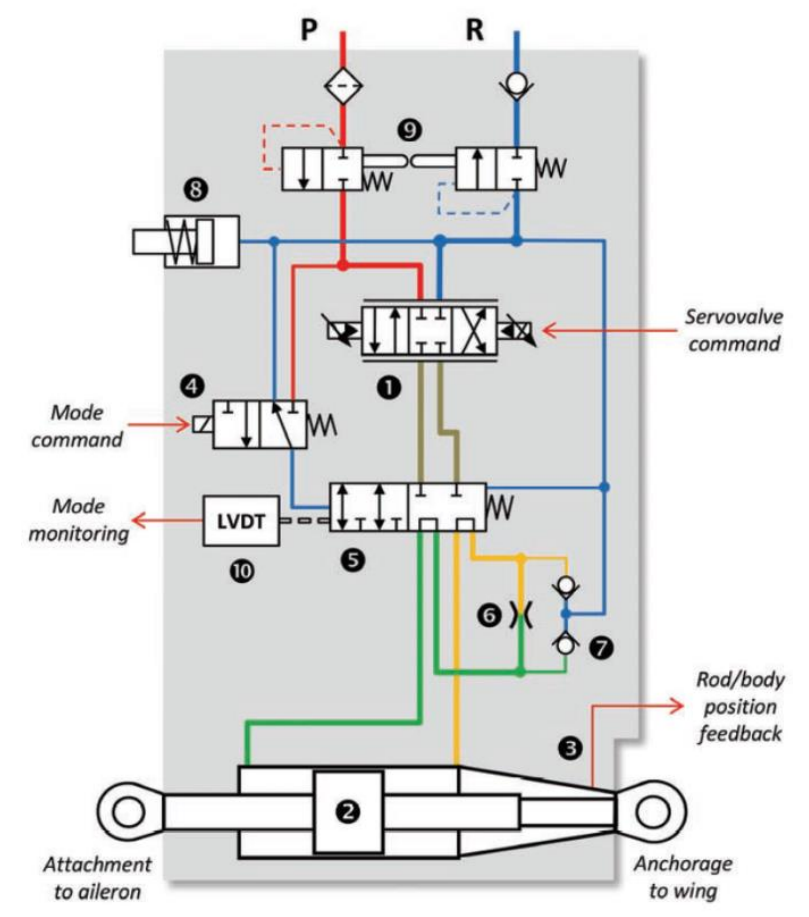

Figure 2. The A320 Aileron Architecture.

In active mode, the aileron actuators' main function is to convert hydraulic power into mechanical power. Such a function can be split into two sub-functions: meter and transform hydraulic power. The components dedicated to performing these two sub-functions are respectively the electro-hydraulic servo-valve (1) and the symmetrical hydraulic cylinder (2); the servo-valve meters the hydraulic power to the cylinder chambers, thus enabling the rod extension or retraction - which is measured by a LVDT sensor (3).

In stand-by mode, both chambers of the cylinder are connected through a hydraulic restriction (6), ensuring the damping of the actuator. In order to avoid the creation of gas in the chambers, anti-cavitation valves (7) are placed so that the return line can provide pressure to the chambers when their pressure becomes too low.

Switching between the two modes is made possible by the mode valve (5), whose position is dependent on its pressurization, controlled by a solenoid valve (4) and measured by a LVDT sensor (10).

Additionally, two mechanically coupled isolation valves (9) are included to keep the fluid inside the actuator in case of pressure supply loss. A fluid compensator (8) enables the storage of low-pressure fluid to compensate for the variation of fluid volume (due to thermal or compressibility effects).

\section{Model-based development workflow of the actuator}

\subsection{Isolation of the actuator sizing}

When breaking down the actuation system function into subsystems, requirements are propagated and refined in each subsystem. A common approach for aircraft manufacturer to do so is to, first, define an aircraft control strategy that distributes the maneuverability requirements to the different flight control surfaces. This applies also for secondary functions, such as load alleviation. It is thus a good first step to assume the actuation of each control surface as isolated and define the performance of each one of these.

The overall problem is obviously more complex and aircraft manufacturers are driven by a mass-reduction objective which requires global optimization of the actuation system. This is beyond the scope of this paper, and we will therefore focus here on a conservative design of an actuator based on known boundary conditions. This will yield a realistic design, not too far from an optimum. This is also a good way to obtain appropriate start values for solving a global optimization problem of the entire system.

A conservative design would assume $70 \%$ of the effective supply pressure available at the actuator hydraulic connectors [SAE12a]. Doing so, the actuator can be sized separately from the rest of the hydraulic system and shall provide back the maximum flow required in order to size the power generation and distribution systems.

\subsection{Actuator requirements and sizing}

Requirement specifications of aerospace actuators contain hundreds of requirements involving performance, constraints, safety, stress, maintainability etc. In order to keep the presentation concise and simple, only a few performance requirements are addressed herein.

The typical characteristics found in the literature [SAE12b] are the maximum load (stall load) of the actuator and maximum rates of the piston (no load speed), in both extension and retraction. These are usually not requirements issued from realistic scenarios but computed based on the design point as these are easy to test on test benches for performance acceptance. For an A320 aileron actuator, these parameters as well as the supply pressure are summarized in Table 1.

In line with the engineering workflow, we choose here to size the actuator directly based on a design point which consists of moving a given load at a given speed. This point is however not available in the literature and thus arbitrarily selected by the authors in order to meet the literature characteristics. This design point is added into Table 1.

Last but not least, control laws require the actuator to have a low phase at low frequencies for small amplitude 
commands. This is covered by the bandwidth requirement of Table 1 .

Table 1 Performance requirement of A320 aileron actuator

\begin{tabular}{|l|l|l|}
\hline \multicolumn{3}{|l|}{ A320 hydraulic system } \\
\hline Supply pressure & $3000 \mathrm{psi}$ & $207 \mathrm{Bar}$ \\
\hline A320 aileron actuator \\
\hline Stroke & $1.7 \mathrm{in}$ & $43 \mathrm{~mm}$ \\
\hline No load speed & $3.5 \mathrm{in} / \mathrm{sec}$ & $89 \mathrm{~mm} / \mathrm{s}$ \\
\hline $\begin{array}{l}\text { Stall load - } \\
\text { Extension }\end{array}$ & $10200 \mathrm{lb}$ & $45.4 \mathrm{kN}$ \\
\hline $\begin{array}{l}\text { Stall load - } \\
\text { Retraction }\end{array}$ & $10200 \mathrm{lb}$ & $45.4 \mathrm{kN}$ \\
\hline $\begin{array}{l}\text { Design point } \\
\text { Bandwidth }\end{array}$ & $\begin{array}{l}72 \mathrm{~mm} / \mathrm{s} \\
20 \mathrm{kN}\end{array}$ \\
\hline
\end{tabular}

\subsection{Design verification and simulator}

Once all parameters have been sized, these are set in the model and simulations are run to verify the current design. These simulations, often called off-design simulations, receives as boundary conditions real command and loading scenarios and compare the output with the expected actuator behavior.

In a following step, the actuator model is typically interfaced with the entire hydraulic system and other consumers, including other actuators, landing gears, etc., and the overall actuation system response is studied.

Finally, the model complexity is reduced, maintaining a satisfactory accuracy in order to export the model for real-time applications - such as flight simulators, for example, for tuning the with flight control laws.

\section{Collaborative development}

In this section, the development of the actuator model described in Section 3 is addressed step by step and collaborative aspects of this process are emphasized. A custom web application developed utilizing the openness of the Modelon Impact tool is also presented. This web application is made to cover the entire development workflow detailed in Section 3.

\subsection{Actuator model assembly}

\subsubsection{Active mode}

In terms of modeling the actuator in active mode, the main components are the symmetric hydraulic cylinder and the electro-hydraulic servo-valve. Both these components are off-the-shelf components in the
Modelon Hydraulics Library, which is the library being used to construct the model. The parameters for the models will come from the sizing performed in Section 4.2.

The hydraulic cylinder model is available as a library component: DoubleActingDualRod. This is a library model that has two variable hydraulic chambers along with the required mechanics such as end stops and mass of the modeled piston. The friction between the cylinder and piston is also modeled. The main required parameter values are the dimensions of the cylinder and piston. While the stroke is a known parameter, the piston and rod areas remain to be defined, at this stage.

The electro-hydraulics servo-valve is modeled using a functional representation which models flow areas between different ports of the servo-valve as variable orifices. The parameters to be provided are the areas of the flow ports.

The supply and return pressures are connected to the servo valve as source and sink. A simple controller is built around the actuator. The controller can command a required position of the piston by controlling the spool position of the servo-valve, as shown in Figure 3.

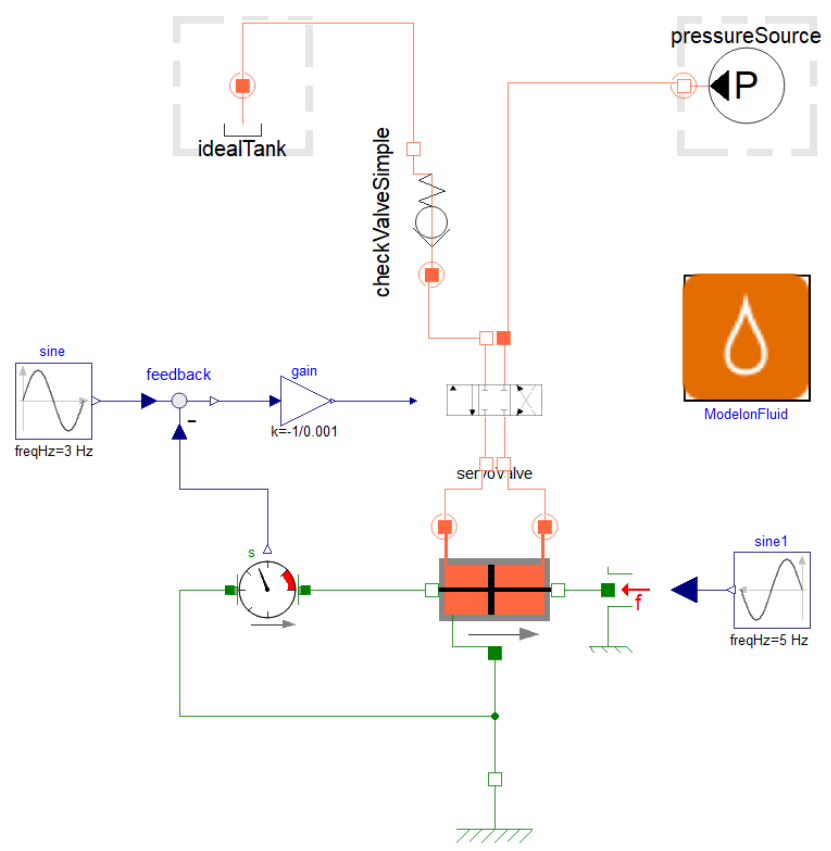

Figure 3. A simple hydraulic actuator model.

\subsubsection{Stand-by mode: the mode valve}

Until now, the A320 aileron actuator model was assembled with all components necessary for simulating the active mode, i.e., in this mode the actuator follows the commands from the Actuator Control Electronic. The next step for increasing the model realism is to add the stand-by (damping) mode and to switch from one mode to the other. This switch is performed by the mode valve.

For the A320 aileron actuator, the mode valve is a six port, two position valves. For out analysis, the anti- 
cavitation valves are removed and thus the mode valve can be simplified to a four ports only. It is positioned between the servo valve and the actuator. Two of the ports are connected to the control ports of the servo valve while the other two are connected to the cylinder chambers.

In active mode, the mode valve establishes connection between the servo valve control ports and the cylinder ports enabling nominal functioning of the system. In stand-by mode, the mode valve isolates the control ports of the servo valve and connects both cylinder chambers through a damping orifice and anticavitation valves - refilling the chambers in case the pressure goes below return pressure.

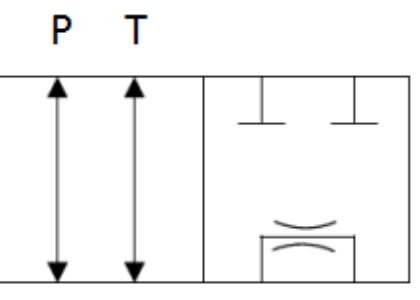

A B

Figure 4. A320 aileron actuator-like mode valve, in a simplified ISO1219 view.

Figure 4 shows an ISO1219 view of this valve, omitting the anti-cavitation valves. Figure 5 shows its location in the actuator model.

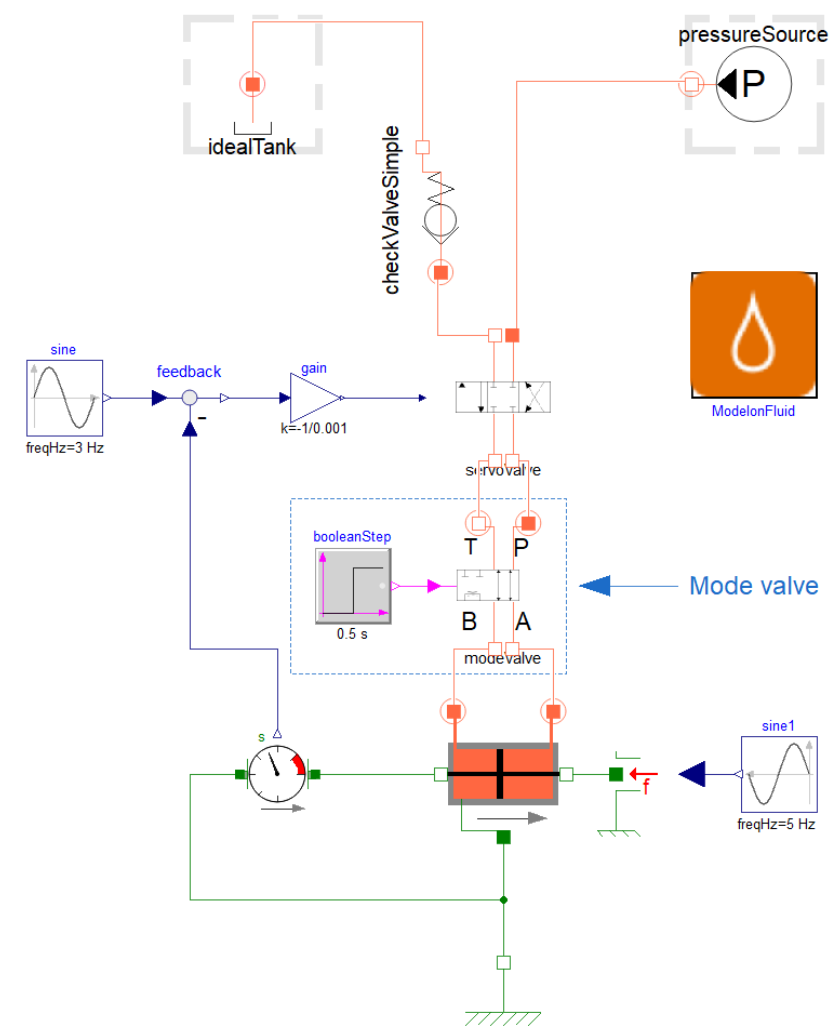

Figure 5. Position of the mode valve in a A320 aileron actuator-like model.

Since all combinations of the directional control valves (DCV) cannot be provided off-the-shelf,
Modelon Hydraulics Library provides a framework in which the user can easily custom-create DCVs of their choice. For the mode valve, a generic DCV with 4 ports and 2 positions can be extended and easily parametrized to fit our needs.

Users need to provide parameters that represents the mapping between the continuous normalized spool position and the normalized flow path opening. In our implementation, the spool position was moved from 0 to 1. The flow path area (normalized) also varies from 0 to 1 where 1 corresponds to the area indicated by nominal flow and pressure drop value provided by the user.

The valve, being generic, assumes all six possible paths - i.e. PA, PB, AT, BT, PT and AB. For the A320 aileron mode valve, the only possible paths are $\mathrm{PA}, \mathrm{BT}$ and $\mathrm{AB}$. The relationship between the spool position and flow path areas can be provided per Figure 6 .

Such mapping between spool position and flow paths can be written in the model through the below parameter assignments. The paths that are not connected do not need to be specified, as their corresponding values default to zero:

$$
\begin{array}{ll}
\text { - } & \text { open_A_B }=[0,0,0.5,1,1] \\
\text { - } & \text { open_B_T }=[1,1,0.5,0,0] \\
\text { - } & \text { open_P_A }=[1,1,0.5,0,0] \\
\text { - } & \text { spool_x_axis }=[0,0.45,0.5,0.55,1]
\end{array}
$$

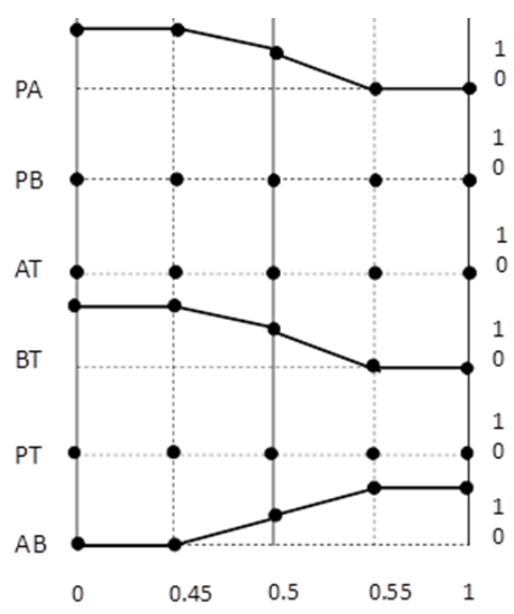

Figure 6. Normalized flow paths v/s spool opening for a mode valve similar to the one of an A320 aileron actuator.

Note that the mode valve is controlled by a unique solenoid valve. This solenoid would provide either the supply or return pressure to the spool surface, moving it to one position or another. In this paper, we decided to only control the valve with a Boolean input to keep it simple.

\subsection{Actuator sizing}

The sizing of the actuator is achieved using the power of Modelica a-causality: boundary conditions are over constrained during initialization while the unknown parameters are relaxed. This way, the model remains 
fully balanced and parameters definition results from the initialization problem solving.

The requirement specification, presented in Table 1, specified steady-state values, exception made of the bandwidth requirement. Consequently, the steady-state initialization, embedded in Modelon Hydraulics library, is selected.

Finally, these performance requirements assume a maximum opening of the valve, which is thus set accordingly in the model.

\subsection{Actuator design verification}

\subsubsection{Speed v/s force characteristic}

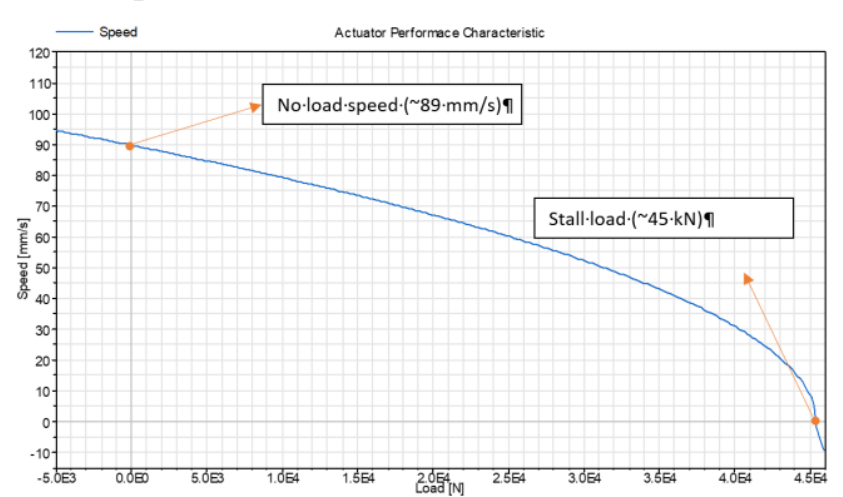

Figure 7. Actuator performance characteristic: speed v/s force.

The design achieved previously can be verified by plotting the speed versus load characteristic of the modeled actuator, we can see that the model meets the actuator requirements of Table 1, in terms of maximum speed and load. A more detailed view is also shown in Figure 11.

\subsubsection{Frequency response}

The frequency domain verification of the actuator is done linearizing the model shown in Figure 3. The Bode plot shown in Figure 8 highlights a close correlation of the bandwidth mentioned in Table 1 .
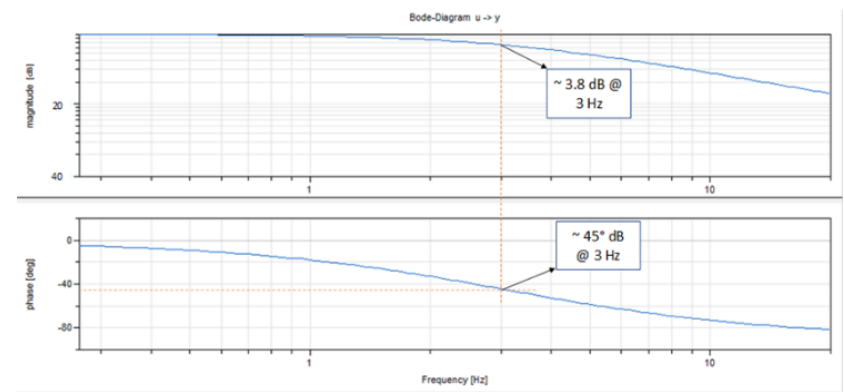

Figure 8. Bode plot showing cut-off frequency.

To achieve these simulations, the parameters were not required to be manually entered by the user as there were computed from on-design simulation and automatically set to the model as off-design parameters - as explained in section 4.2.

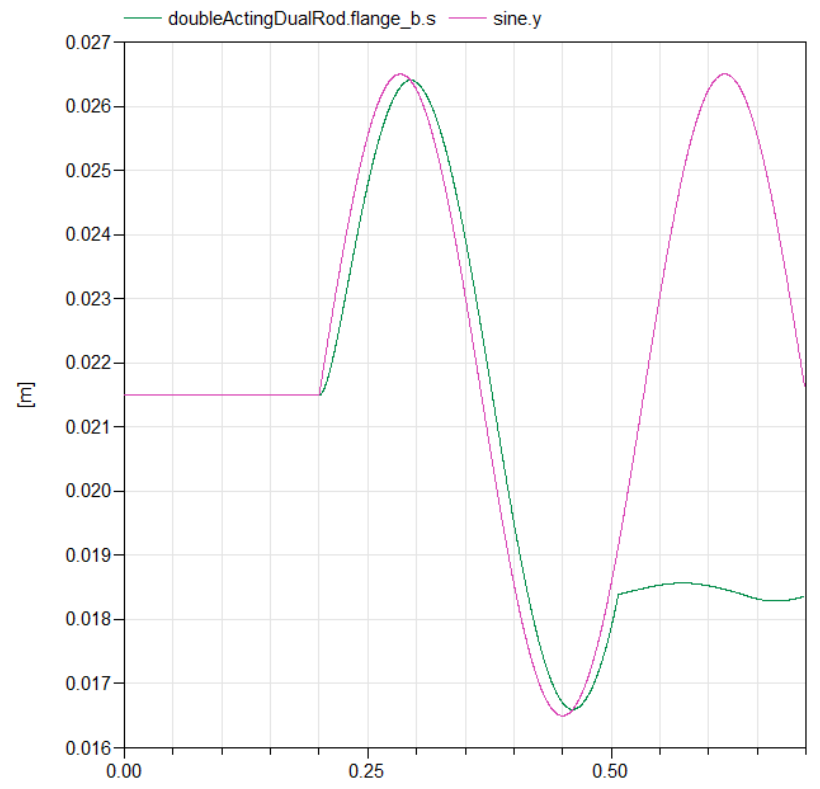

Figure 9. Active/stand-by switch mode at time 0.5 .s

\subsubsection{Time and fault response}

In order to illustrate the mode switching, a dynamic simulation scenario is run where the actuator is initially in active mode and at time 0.5 it switches to the damping mode. The actuator is exposed to a sine antagonist load which is easily faced by the hydraulic actuator in active mode but that drives the actuator in by-pass mode.

\subsection{Web Application}

Modelon Impact enables users to create and run their own web-applications. Through Python REST API, Modelon Impact allows communication between the web-app and the models. To provide even more flexibility, Python or JavaScript scripting is allowed. This gives the possibility to implement complex workflow into an easy flowing webpage that can be used by many engineers who do not want to get into the details of modeling parts.

A dedicated web-application was developed to summarize the entire workflow presented until now in this paper.

\subsubsection{Performance requirements}

The performance requirements of the actuator are fed into the app through discrete data points that the actuator must be satisfied in terms of actuator speed and load points. A specific Python script was developed and integrated in the web-application to determine the more constraining points and draw an enclosing curve that will be the envelope for the actuator performance as shown in Figure 10. Points below this enclosing curve would no be relevant to investigate for sizing purpose. 


\section{Modelon_ Actuator Design WebApp}

\section{Mission Profile}

Please choose a file containing load $\mathrm{v} / \mathrm{s}$ speed mission profile of the actuator to be sized. Choose File ScatteredData.csv

The scattered data can be visualized in the below graph. By pressing the fit button fit, the more constraining points are selected and the enclosing curve displayed.

\section{Force vs Speed}

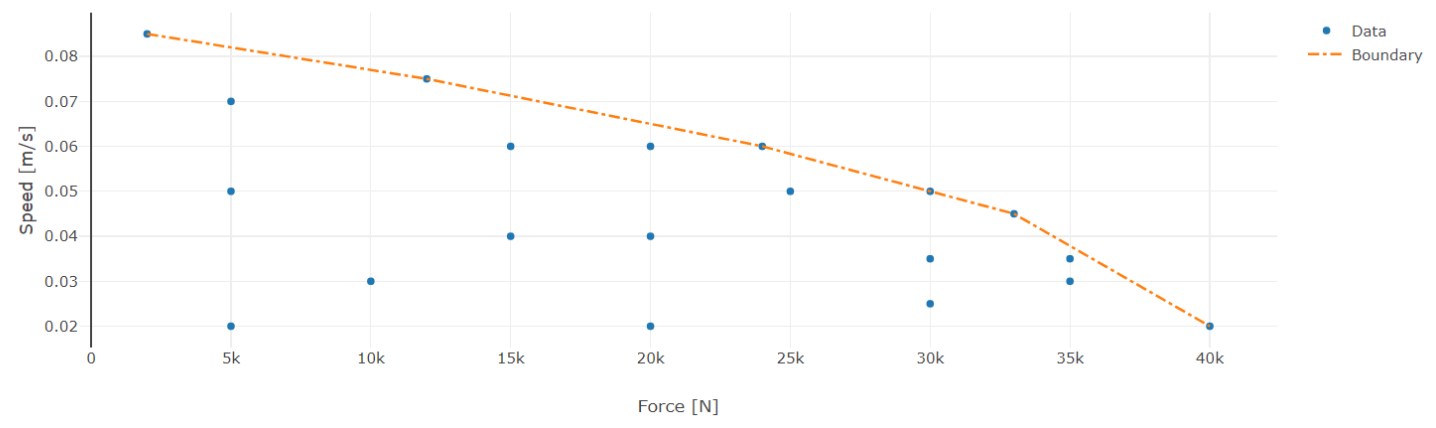

Figure 10. WebApp - view of the performance requirements with the enclosing curve.

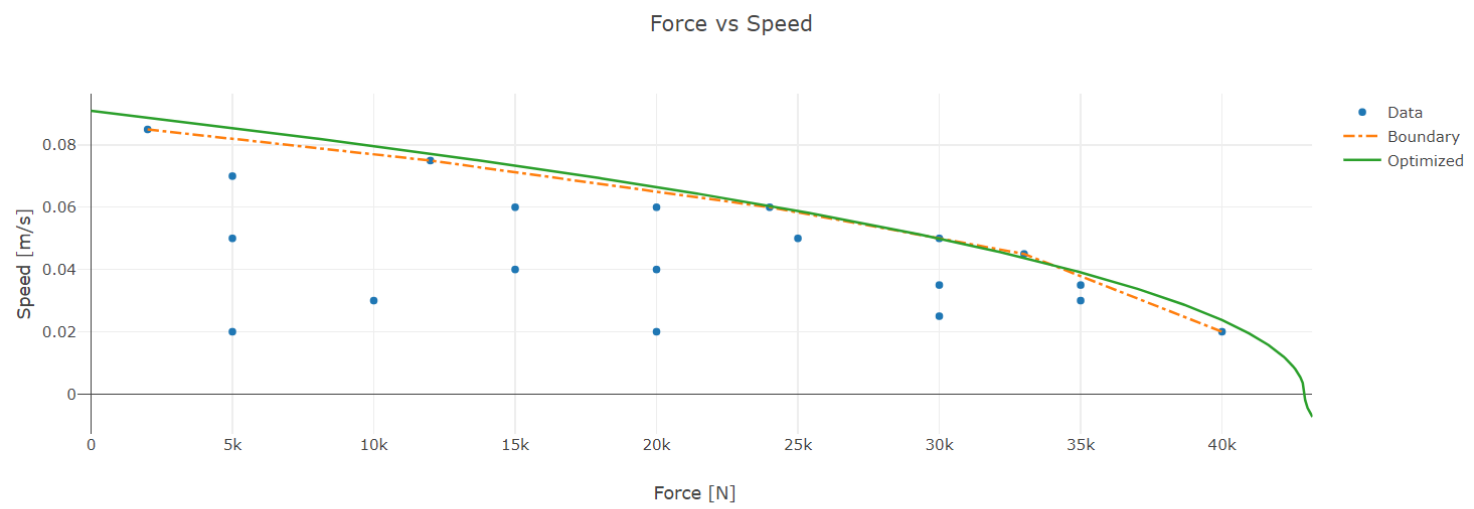

Parameters \& Optimization
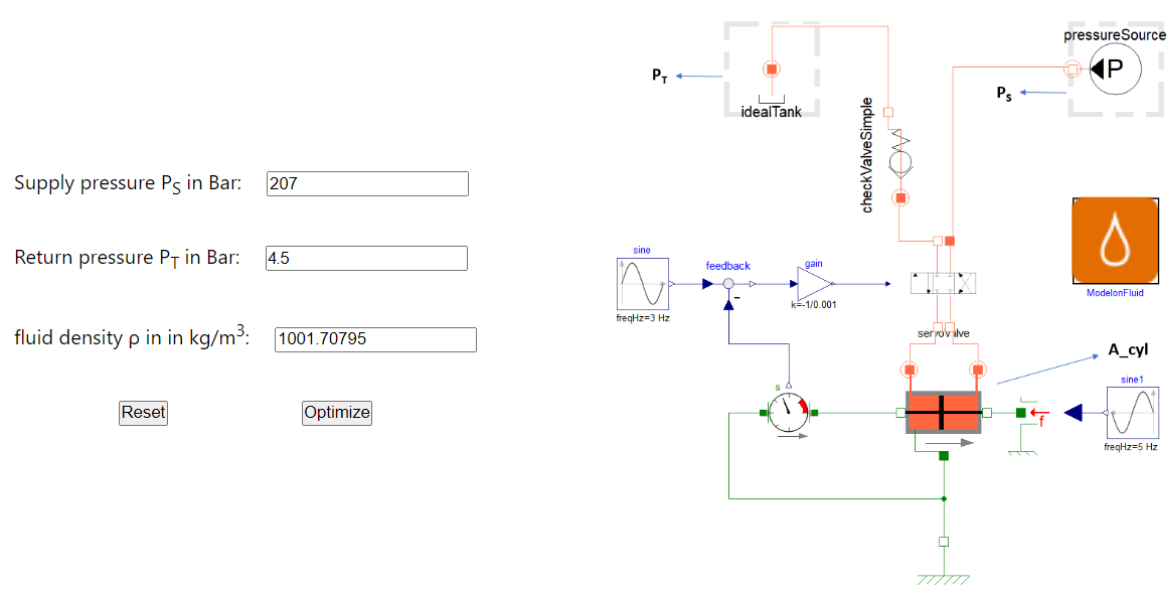

Figure 11. WebApp - optimized design based on parameter inputs for boundary conditions. 


\subsubsection{Actuator sizing}

The next step is to provide the boundary conditions of the actuator system under which the desired performance must be achieved. These are the system boundary pressures and hydraulics fluid density. A simple model is used by the WebApp to run the different designs (actuator cylinder area) through an optimization routine that provides a design which satisfies the enclosing curve of the envelope. This is shown clearly in the Figure 11.

\subsubsection{Actuator analysis}

The final step of this workflow is to apply the design to the complex dynamic model of the actuator system and perform frequency analysis. The parameters computed previously are applied to the complex model automatically (action performed by the web-application through Modelon Impact API) and state space representation is extracted by the WebApp. This in turn is used to get the bode plots for the actuator control system in totality as shown in Figure 12.

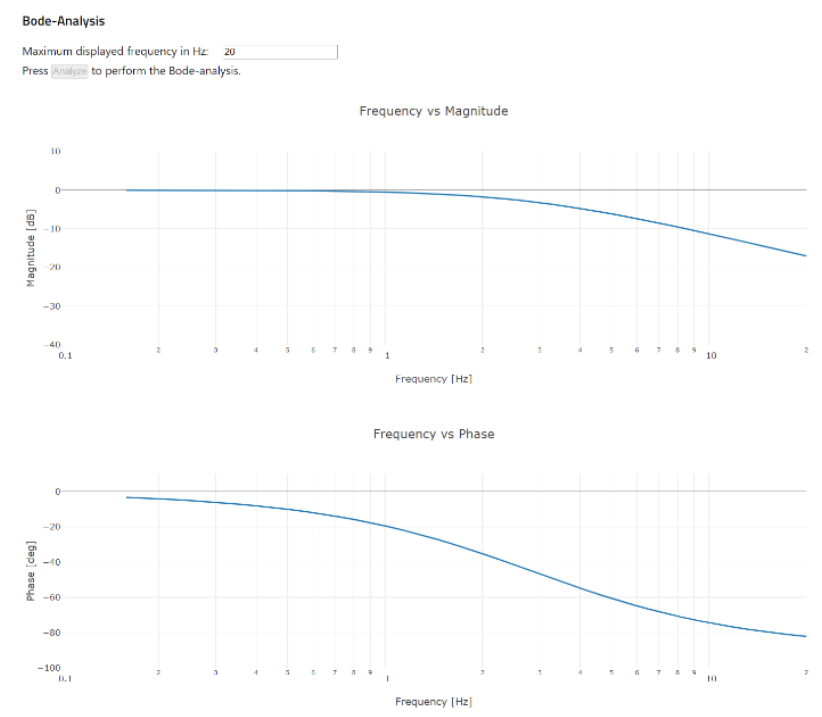

Figure 12. WebApp showing the Bode plot of the actuator control system.

\subsubsection{Different user types}

Modelon Impact brings a lot of value to a company, even more, in combination with web-applications. While with the graphical user interfaces, the extensive set of libraries, the state of the art compiler and FMI support Modelon Impact opens the door to simulation to many users, the web-applications enable all remaining persons to consume the models, based on a given workflow.

\subsection{Real-time simulation}

During the development of a Fly-by-Wire actuator and its control electronics, tuning the actuator control laws is of particular importance. While most can be performed by simulation on a standard computer, one relevant step is to test the control laws when executed on a real-time target, which in turn controls the physical plant model, i.e., the hydraulic actuator, inputs. These two models are usually not coming from the same software.

It is critical that the plant model can be simulated in real-time i.e., the computation of the model outputs consumes less time than the corresponding simulated time step. If this is not the case, overruns result which will render incorrect results of the simulation. This type of real-time integration enables validating the actual actuator control laws under more realistic conditions.

In the following, we will illustrate how the actuator model developed in Section 5.1 is integrated in a Speedgoat target and runs real-time.

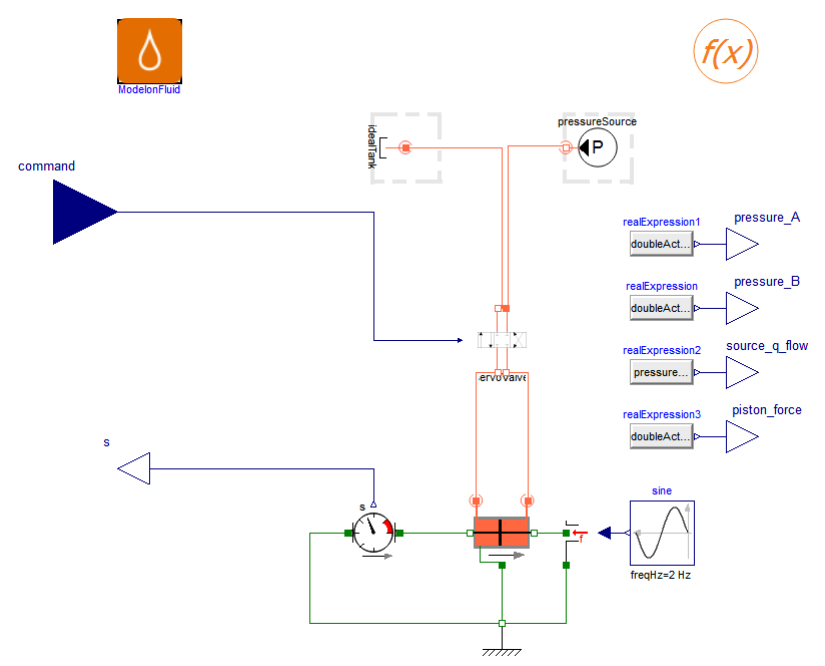

Figure 13. The actuator with interfaces for FMU export.

\subsubsection{Model adaptations for HiL simulation}

The changes in order to achieve real-time performance are the following:

- The mode valve is removed. The intent of the HiL simulation is to observe the actuator response to commands, meaning the stand-by mode would bring only complexity that is not needed.

- Isolation of the plant model, since the control logic will be in the real-time target implemented in Matlab Simulink®.

- Addition of a outputs monitoring the pressures, flow rate and force of the plant model, to provide easy access and and plotting of these.

Figure 13 displays the final version of the model.

\subsubsection{Integration on the Real-Time target}

The integration of the model in the Real-Time target consists of a few steps:

- Encoding in Simulink ${ }^{\circledR}$ of the control/command blocks for the actuators. 
- Inclusion of the Modelica model made from Modelon Hydraulics, exported as an FMU, using FMI Toolbox for MATLAB/Simulink ${ }^{6}$.

- Adding Speedgoat-specific blocks to monitor the variables of interest.

- Build the real time executable using the Simulink Real-Time ${ }^{\mathrm{TM}}$ target.

Figure 14 shows the result of the first three steps listed above, prior to generation of the executable.

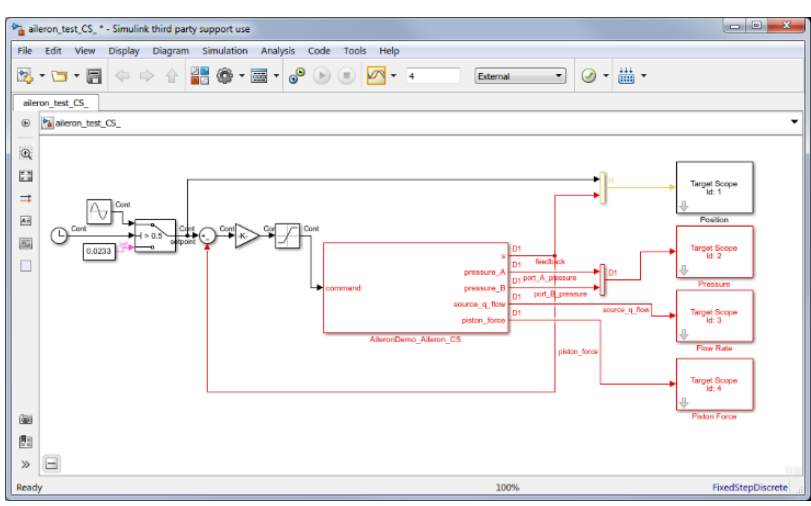

Figure 14. The actuator plant model, implemented in Simulink for import to the Speedgoat real-time target.

In this application, the plant model has been exported with its own solver (FMU for co-simulation) so that both the control logic and the plant model have separate solvers and the models communicate at each time step.

\subsubsection{Real-time simulation of the integrated system}

Once the model is integrated and imported on the realtime target, it satisfies hard real-time requirements. The main variables set as outputs previously are plotted in Figure 15:

- Top left: actuator command and response. We observe an initial position of the actuator onpurpose different from the command leading to a fast transient at the beginning of the simulation. After reaching the setpoint, the actuator reacts in a satisfactory dynamic behavior, slightly influenced by the antagonist loads.

- Bottom left: the flow exiting the ideal pressure source is plotted. The dynamic observed is a redressed sine similar to the actuator position response. The frequency is doubled as the flow is provided in both positive and negative speeds of the actuator.

- Top right: the pressure in both chambers are observed. These are oscillating around an average point, in phase with the force oscillation shown at the bottom right.

Note: to avoid having a larger black picture, contrast on Figure 15 have been changed.

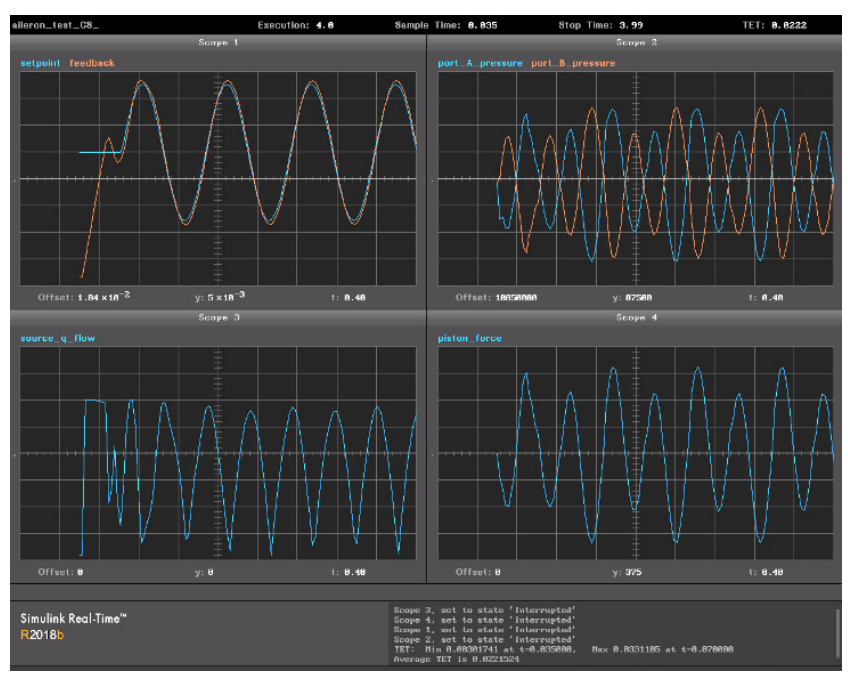

Figure 15. Plots of the main variables of interests in the Speedgoat GUI.

\section{Summary and conclusions}

This paper addressed the engineering workflow of model-based development of an aircraft hydraulic actuator. In order to be realistic, the focus was led on the need to enable a collaborative development - which involved several users and several tools. Modelon Impact was introduced as one of these and brought a new dimension when spreading the model usage to a relatively broad audience through relevant web-apps. Another key aspect for this collaborative development were the availability of Modelon Hydraulics library in several platform and Modelon tools to support FMI standard.

\section{References}

[COI16] Coïc, C., Model-Aided Design of a HighPerformance Fly-by-Wire Actuator, Based on a Global Modelling of the Actuation System using Bond-graphs. PhD dissertation, INSA Toulouse/Airbus Helicopters, 2016.

[DRE14] Drenth, E., Törmänen, M., Johansson, K., Andersson, B.-A., Andersson, D., Torstensson, I. \& Åkesson, J., Consistent Simulation Environment with FMI based Tool Chain, The $10^{\text {th }}$ International Modelica Conference, Lund, Sweden, 2014.

[MAR18] Maré, J.-C., Aerospace Actuators 3: European Commercial Aircraft and Tiltrotor Aircraft. ISTEWiley, 2018.

[SAE12a] SAE Aerospace, AIR994 - Recommended Practice for the Design of Tubing Installations for Aerospace Fluid Power Systems. USA, 2012.

[SAE12b] SAE Aerospace, AIR4253B - Description of Actuation Systems for Aircraft With Fly-By-Wire Flight Control Systems. Aerospace Information Report, 2012.

\footnotetext{
${ }^{6}$ https://www.modelon.com/products-services/modelondeployment-suite/fmi-toolbox/
} 